\title{
Cultura organizacional de enfermería dominante en un hospital de la Secretaría de Salud de Morelos, México
}

\author{
Doris Verónica Ortega-Altamirano, M en C, (I) María de Lourdes Alemán-Escobar, M en E Sup, ${ }^{(1)}$ \\ Ivone Brito-García, EESP,(2) Celina Magally Rueda-Neria, LI, (') Rey Arturo Salcedo-Álvarez, MSP.(3)
}

\begin{abstract}
Ortega-Altamirano DV,Alemán-Escobar ML, Brito-García I, Rueda-Neria CM, Salcedo-Álvarez RA. Cultura organizacional de enfermería dominante en un hospital de la Secretaría de Salud de Morelos, México.
\end{abstract} Salud Publica Mex 20I I;53:1 I-16.

\begin{abstract}
Resumen
Objetivo. Identificar los modelos de cultura organizacional, dominante y preferido, en enfermería en el segundo nivel de atención a la salud. Material y métodos. Entre abril y junio de 2008 se hizo un estudio transversal con personal de enfermería de los Servicios de Salud del Estado de Morelos. Se obtuvo información sobre los datos sociodemográficos y la cultura organizacional. Se hicieron pruebas de $\mathrm{ji}^{2}$ y de correlación Kappa, y se usó Stata v. IO. Resultados. Se entrevistó a 30 enfermeras seleccionadas aleatoriamente de un total de 397 , con al menos un año de antigüedad laboral. Se identificó que la cultura organizacional dominante es la del poder y la preferida es la del desempeño. El coeficiente Kappa positivo de $0.17(p=0.047)$ entre lo que domina y el modelo del desempeño que se prefiere. Conclusiones. Identificar estos modelos de la cultura organizacional permite entender los diferenciales de desempeño en la gestión del cuidado enfermero a los usuarios hospitalarios y plantearse aspiraciones de mejoras en el propio servicio.
\end{abstract}

Palabras clave: servicios de salud; cultura organizacional; gerencia; servicios de enfermería; México
Ortega-Altamirano DV,Alemán-Escobar ML, Brito-García I, Rueda-Neria CM, Salcedo-Álvarez RA.

Predominant organizational culture in nursing at a public hospital. Morelos, Mexico.

Salud Publica Mex 20I I;53:II-16.

\begin{abstract}
Objective. To identify dominant and preferred organizational culture models at second level nursing services in Morelos. Material and Methods. A cross-sectional study was conducted between April and June, 2008. Nursing caregivers from Public Health Services of Morelos State participated. We gathered and analyzed data about socioeconomic characteristics and organizational culture, and we applied the $X^{2}$ and Kappa coefficient statistical tests using Stata version 10 software. Results. We interviewed 30 nurses, randomly selected from a total of 397 , with at least one year of seniority. It was identified that the dominant organizational culture is one of power and the preferred culture is one of performance. A Kappa positive coefficient of $0.17(p=0.047)$ was found between the dominant model and the preferred performance model. Conclusions. Identifying said organizational culture models helps to understand performance differences in the management of nursing care services currently provided to users of the hospital and to aspire to improvements in the service itself.
\end{abstract}

Key words: organizational culture; nursing services; administration; management; Mexico

(I) Centro de Investigación en Sistemas de Salud, Instituto Nacional de Salud Pública. Cuernavaca, Morelos, México.

(2) Centro de Investigación en Salud Poblacional, Instituto Nacional de Salud Pública. Cuernavaca, Morelos, México.

(3) Unidad de Investigación, Escuela Nacional de Enfermería y Obstetricia,Universidad Nacional Autónoma de México. México DF, México.

Fecha de recibido: 3 de marzo de 2010 - Fecha de aceptado: 25 de noviembre de 2010

Solicitud de sobretiros: M en E. Sup. María de Lourdes Alemán Escobar.Av. Universidad 655. Col. Santa María Ahuacatitlán.

62100 Cuernavaca, Morelos, México.

Correo electrónico: lourdes.aleman@insp.mx 
$\mathrm{E}_{\mathrm{t}}^{\mathrm{n}}$ México, el Sistema Nacional de Salud (SNS) actualmente se enfrenta a la necesidad de responder, con efectividad, a los cambios epidemiológicos de la población. Algunos de los cambios epidemiológicos se dan en enfermedades como la diabetes mellitus, que en 1993 tuvo una prevalencia de $4.6 \%$ y que para 2006 fue de $23.8 \% .^{1}$ Otro ejemplo es la hipertensión arterial diagnosticada en 26 millones de personas en el año 2000, y con un estimado de 1.3 hipertensos no diagnosticados. ${ }^{2}$

La efectividad de la respuesta del SNS a las necesidades de la población depende de la organización de los servicios de salud, principalmente de los recursos humanos y financieros. Arredondo y colaboradores analizan la respuesta del SNS a ambos padecimientos en términos financieros y concluyen que la demanda de atención hospitalaria tiende a incrementarse. ${ }^{1-2}$

Para la Secretaría de Salud es imperativo ampliar la cobertura y mejorar la calidad en el campo asistencial ${ }^{3}$ conforme a las Reglas de Operación 2010 del Programa Sistema Integral de Calidad en Salud (SICALIDAD). ${ }^{4}$ Sin embargo, aumentar el número de recursos humanos en hospitales no es suficiente pues debe asegurarse su óptimo desempeño en los aspectos técnicos y operacionales. En términos organizacionales, gran parte de la efectividad de la atención depende del desempeño de sus recursos humanos.

Los rasgos de la cultura organizacional en el ámbito hospitalario se identifican a través de los procesos internos de toma de decisiones, las formas de comunicación, de los criterios de avance de los profesionales y satisfacción laboral del personal. También se identifica por los valores, creencias y actitudes que rigen el desempeño y el logro de los resultados de la organización. ${ }^{5}$

La cultura organizacional en los hospitales está condicionada por múltiples aspectos como el marco jurídico que soporta las condiciones laborales y contractuales del personal; la misión y la visión de la organización, la estructura organizacional vigente, así como las políticas y las formas de financiamiento y la cultura predominante en el contexto social. ${ }^{6}$

Entre más independencia alcanzan los distintos servicios que conforman a los hospitales, es mayor su eficacia para responder a la demanda. ${ }^{7}$ Este estudio se enfoca a los servicios de enfermería por ser medulares en la estructura organizacional hospitalaria.

Kurganct y colaboradores, ${ }^{5}$ en 2008, reportan que los rasgos de la cultura organizacional moderan el desempeño del personal de enfermería y la efectividad de la gestión de los servicios de salud en Brasil.

En 2009, Ng TWH y colaboradores ${ }^{8}$ enfatizan que la satisfacción laboral y el desempeño en el trabajo se correlacionan positivamente en tres contextos culturales distintos pero con rasgos similares: el poder y la autonomía son análogos entre todos sus miembros y los riesgos e incertidumbre son escasos.

Schepers, en 2006, ${ }^{9}$ compara dos modelos de cultura organizacional en hospitales belgas y reporta que su efectividad es diferencial. Hay mejores resultados en el hospital en el que la cultura se caracteriza por el balance entre lo gerencial y lo profesional, tanto en personal médico como en el de enfermería.

Exponentes como Fleury, Freitas, Harrison y Handy ${ }^{10}$ han contribuido a la difusión y al entendimiento de diversos modelos de cultura organizacional y han aportado algunos métodos para la caracterización de los modelos de cultura organizacional. Se define a los modelos de cultura organizacional como el conjunto de reglas, símbolos, creencias y valores compartidos y adquiridos de manera voluntaria o inconsciente por todos los miembros de una organización o grupo humano, que le proporcionan la cohesión necesaria para trabajar armónicamente hacia la consecución de los objetivos comunes de la institución. ${ }^{11}$

Desde la perspectiva de los modelos de ciclo de vida de las empresas, el grado de desarrollo de la cultura de una organización se expresa por el modelo dominante y la influencia que éste ejerce en los procesos de gestión. Por grado de autonomía, los modelos son clasificados como el del poder; el de la función o tarea; el del desempeño; $y$, del apoyo. ${ }^{12}$

Del año 1996 al 2000, Hartasánchez probó los modelos de cultura organizacional del ciclo de vida de las empresas en el ámbito público para identificar la cultura prevaleciente y la deseada en organizaciones públicas de México. Reportó que los modelos de cultura organizacional predominantes son los del poder y la función. ${ }^{12}$

Las características que comparten ambos modelos son que marcan las distancias entre los estratos sociales, la baja motivación para correr riesgos, resistencia a las innovaciones institucionales e imperan los valores masculinos, por ejemplo, roles activo o pasivo, atribuidos a masculino y femenino, respectivamente, propios de estructuras burocráticas que surgen de los procesos de institucionalización que existen en nuestro país desde hace más de siete décadas.

El instrumento desarrollado por Hartasánchez para el ámbito de la administración pública de México fue retomado, adaptado y probado por Alemán Escobar y colaboradores en los servicios de enfermería en el primer nivel de atención, en el año $2004 .^{13}$

El objetivo de este estudio es identificar los modelos de cultura organizacional, dominante y preferido en personal de enfermería dadas sus creencias, actitudes, valores y comportamientos, en un hospital público del segundo nivel de atención a la salud en el estado de Morelos, México. 


\section{Material y métodos}

Entre abril y junio de 2008 se aplicó en un hospital del segundo nivel de atención de la Secretaría de Salud del Estado de Morelos, México, un cuestionario para caracterizar la cultura organizacional de los servicios de enfermería.

Aleatoriamente se eligieron 34 participantes de los 397 que cumplieron con los criterios de selección: tener una antigüedad laboral de un año o más y estar adscrito al servicio de enfermería del hospital. El tamaño de la muestra se calculó con base en estimaciones hipotéticas de la prevalencia de $90 \%$ para las creencias, actitudes y conductas relativas a la cultura organizacional; el nivel de confianza usado fue de $95 \%$ y el de precisión de $0.10 \%$.

El cuestionario constó de diez variables y una sección de datos generales. Para cada variable se disponía de cuatro respuestas. Las respuestas aluden a creencias, actitudes y conductas propias de cada uno de los cuatro modelos de cultura organizacional antes descritos. Las variables fueron: 1) elección de prioridades del personal dentro del servicio; 2) creencias sobre lo que hace a las personas contar con la consideración de los colegas; 3) actitud respecto al trato que recibe el personal por parte de la institución; 4) creencias sobre lo que influye en el desempeño del personal de enfermería; 5) creencias acerca de los procesos de toma de decisiones por parte del personal de enfermería; 6) actitud y conducta hacia la asignación de tareas o puestos de trabajo;7) creencias sobre las expectativas del personal de enfermería por parte de personal externo al servicio; 8) creencias sobre las expectativas del personal de enfermería por parte de directivos y otras autoridades; 9) actitud hacia recibir indicaciones por parte de los compañeros del mismo servicio; $y, 10)$ actitud hacia el trabajo.

Los datos recopilados fueron ponderados y se conformó la variable dependiente en cuatro categorías; cada una corresponde a cada tipo de modelo de cultura organizacional. Las mismas categorías se utilizaron en dos circunstancias: lo que predomina en la institución y lo deseable. Las proporciones de ambas circunstancias fueron comparadas con el estadístico $\mathrm{ji}^{2}$ y el coeficiente kappa. Para procesar la información se utilizó el programa de análisis estadístico Stata v.10.

Tres enfermeras inscritas en un programa de posgrado en enfermería en salud pública fueron capacitadas para llevar a cabo la aplicación de los cuestionarios. Se aseguró la confidencialidad, el anonimato y la privacidad a los participantes al contar con un lugar individual para responder el cuestionario.

Se obtuvo el consentimiento informado verbal de cada participante antes de entregarle el cuestionario. Se les comunicó que el estudio contó con la autorización de las Comisiones de Ética e Investigación del hospital sede del estudio.

\section{Resultados}

Para el personal de los servicios de enfermería, la cultura organizacional mostró que el modelo del poder es lo prevaleciente y su ideal es el modelo del desempeño. El modelo del poder, aunque es el mayormente experimentado como dominante, es también el más rechazado. Como preferencia de modelo de cultura organizacional, se señala discretamente al modelo del desempeño.

El cuadro I muestra las características sociodemográficas encontradas en los participantes $(n=30)$. Respecto a la edad, el rango mayoritario fue el de 31 a 40 años, $46.6 \%$ ( $n=14)$. Sólo dos participantes fueron menores de 30 años de edad, $6.7 \%$ ( $n=2)$ al momento del estudio.

\section{Cuadro I \\ ENFERMERAS SEGÚN ESCOLARIDAD, CATEGORÍA LABORAL y antigüedad, Hospital General José G. Parres, Secretaría de Salud del estado de Morelos, MéxIco 2008}

$$
\begin{gathered}
\text { Frecuencia } \\
n=(30)
\end{gathered} \quad \%
$$

Edad en años

\begin{tabular}{rrr}
$20-30$ & 2 & 6.7 \\
\hline $31-40$ & 14 & 46.6 \\
\hline $41-50$ & 9 & 30 \\
\hline$\geq 51$ & 5 & 16.7
\end{tabular}

Escolaridad

\begin{tabular}{lrl} 
Técnica & 23 & 76.7 \\
\hline Bachillerato & 4 & 13.3 \\
\hline Licenciatura & 3 & 10
\end{tabular}

Categoría laboral

\begin{tabular}{lrl} 
Enfermera auxiliar & 8 & 26.7 \\
\hline Enfermera general & 10 & 33.3 \\
\hline Enfermera especialista & 6 & 20 \\
\hline Supervisora y subjefa & 6 & 20
\end{tabular}

Antigüedad en años

\begin{tabular}{lcl} 
I a 10 & 6 & 20 \\
\hline$\geq I 1$ & 24 & 79.9
\end{tabular}

Fuente: Cuestionario de diagnóstico de cultura organizacional de los servicios de enfermería aplicado en el Hospital General José G. Parrés. Cuernavaca, Morelos, 3, 8, 15 y 17 de abril de 2008 
En cuanto a la escolaridad, $77 \%(\mathrm{n}=23)$ de los participantes cuenta con estudios a nivel técnico y uno de cada 10 participantes tiene el nivel de licenciatura, con $10 \%(\mathrm{n}=3)$. Acerca de la categoría laboral, 33.3\% $(\mathrm{n}=10)$ son enfermeras y enfermeros generales, $26.7 \%(\mathrm{n}=8)$ son auxiliares y $20 \%(\mathrm{n}=6)$ especialistas. La antigüedad laboral de los participantes fue mayor a los 11 años en $80 \%(\mathrm{n}=24)$ de los entrevistados y de éstos, en 10 participantes la antigüedad fue mayor a los 20 años.

El cuadro II presenta los modelos de cultura organizacional reportados por el personal de enfermería que participó en el estudio. Sobresale como dominante el modelo del poder en la cultura organizacional, con $43.3 \%$ ( $n=13)$; en cambio, en la misma proporción fue

\section{Cuadro II}

MOdelo de CULTURA ORGANIZACIONAL DOMINANTE VS PREFERIDO POR EL PERSONAL DE ENFERMERÍA Hospital General José G. Parres; Secretaría de Salud del Estado de Morelos, México 2008

\begin{tabular}{|c|c|c|c|c|}
\hline Modelo & Dominante & Preferido & Kappa & $p P$ \\
\hline \multicolumn{5}{|l|}{ Poder } \\
\hline \multicolumn{5}{|l|}{ Sí } \\
\hline$\%$ & 43.33 & 6.67 & \multirow{5}{*}{0.17} & \multirow{5}{*}{0.047} \\
\hline$n$ & 13 & 2 & & \\
\hline \multicolumn{3}{|l|}{ No } & & \\
\hline$\%$ & 56.67 & 93.33 & & \\
\hline $\mathrm{N}$ & 17 & 28 & & \\
\hline \multicolumn{5}{|l|}{ Función } \\
\hline \multicolumn{5}{|l|}{ Sí } \\
\hline$\%$ & 30.0 & 20.0 & \multirow{5}{*}{-0.31} & \multirow{5}{*}{0.96} \\
\hline $\mathrm{n}$ & 9 & 6 & & \\
\hline \multicolumn{3}{|l|}{ No } & & \\
\hline$\%$ & 70 & 80.0 & & \\
\hline $\mathrm{n}$ & 21 & 24 & & \\
\hline \multicolumn{5}{|l|}{ Desempeño } \\
\hline \multicolumn{5}{|l|}{$\mathrm{Si}$} \\
\hline$\%$ & 16.6 & 43.33 & \multirow{5}{*}{-0.31} & \multirow{5}{*}{0.98} \\
\hline $\mathrm{n}$ & 5 & 13 & & \\
\hline \multicolumn{3}{|l|}{ No } & & \\
\hline$\%$ & 83.33 & 56.67 & & \\
\hline$n$ & 25 & 17 & & \\
\hline \multicolumn{5}{|l|}{ Apoyo } \\
\hline \multicolumn{5}{|l|}{$\mathrm{Si}$} \\
\hline$\%$ & 10.0 & 30 & \multirow{5}{*}{-0.17} & \multirow{5}{*}{0.88} \\
\hline $\mathrm{n}$ & 3 & 9 & & \\
\hline No & & & & \\
\hline$\%$ & 90.0 & 70.0 & & \\
\hline$n$ & 27 & 21 & & \\
\hline
\end{tabular}

Fuente: Cuestionario de diagnóstico de cultura organizacional de los servicios de enfermería, aplicado en el Hospital General José G. Parres, Cuernavaca, Morelos. 3, 8, 15 y 17 de abril de 2008 preferido el modelo del desempeño, 43\% (n=13). Entre los modelos ideales, el modelo del desempeño presenta una proporción de aceptación de 43.3\% (n=13); la preferencia no es estadísticamente significativa.

Al comparar los modelos de cultura organizacional, se encontró que su correlación es negativa entre el del poder y los del apoyo, la función y el desempeño, con coeficientes kappa de $-0.17,-0.31$ y -0.31 , respectivamente. El modelo del poder presentó un coeficiente kappa positivo, $0.17(p=0.047)$ entre lo que domina y el modelo del desempeño, que se prefiere.

El modelo del poder es rechazado por $93.33 \%(n=28)$ de los participantes, $p=0.047$. Entre los modelos más rechazados están los de la función y del apoyo, con $80 \%$ $(n=24)$ y $70 \%(n=21)$, respectivamente.

La utilidad de estos resultados radica en que se analizaron los datos obtenidos en el marco del contexto institucional de la Secretaría de Salud, lo que documenta, en la práctica, las recomendaciones para la mejora de los procesos de gestión de los servicios de enfermería en hospitales de segundo nivel de atención.

\section{Discusión}

Se identificó como el modelo de cultura organizacional dominante al del poder, lo que es consistente con lo reportado por Hartasánchez en el año 2002 y por AlemánEscobar y colaboradores en el año 2005, para el primer nivel de atención, también en servicios de enfermería de la Secretaría de Salud. ${ }^{12,13}$

Este hallazgo implica que en la institución estudiada existe la reticencia a las innovaciones o las mejoras de los procesos administrativos y de gestión de los servicios de enfermería. Como lo reporta $\mathrm{Ng}$ en el $2008,{ }^{8}$ a pesar de que los resultados del servicio no sean satisfactorios ni para la institución ni para el propio personal, se perpetúan los procedimientos y normas tradicionales.

El hecho de que sea este el modelo dominante confirma que en la gestión de los cuidados enfermeros no hay cabida a la iniciativa, la creatividad ni a la autonomía del personal. También marca las distancias entre profesionales de la salud del área de la enfermería., ${ }^{9}, 2$

El modelo del poder es unánimemente rechazado por los enfermeros y las enfermeras. Los rasgos de la cultura organizacional que le son propicios son dos, uno que es atribuible al personal y el otro a la institución. Respecto al personal, su perfil de formación técnica carece de competencias profesionales y laborales necesarias para un alto desempeño. Respecto a la institución, ésta es estable y con pocos cambios en sus normas, lo que dificulta la introducción de innovaciones gerenciales coherentes con los cambios de las crecientes y complejas necesidades. 
En el estudio llevado a cabo con este personal en el municipio de Sao Paulo, Brasil, se observó que el estancamiento del desarrollo profesional de enfermería y su escasa o nula participación en la toma de decisiones son obstáculos para la satisfacción laboral y aumentan el ausentismo. ${ }^{9}$ Se observó insuficiente cumplimiento de las metas, escasa capacidad resolutiva de los casos y desperdicio de los recursos materiales y humanos.

La cultura organizacional basada en el poder está presente en la totalidad de la institución, tanto en la estructura como en el funcionamiento. Esto fue señalado previamente por Carvalho-Jericó y colaboradores, en $2008 .^{14}$

El grupo de enfermeras estudiadas prefirió el modelo de cultura del desempeño, seguido muy de cerca por el del apoyo. El modelo del desempeño tiene la ventaja de incrementar la productividad y la eficiencia en los servicios de enfermería. Estos resultados son similares a los reportados por Hartasánchez como los preferidos o anhelados por los líderes, población que él ha estudiado, ${ }^{12}$ y ambos tienen en común los modelos del desempeño y del apoyo, que favorecen la iniciativa propia del personal y su creatividad asi como el énfasis en la colaboración solidaria y en la autonomía de los profesionales.

Se entiende la preferencia por el modelo del desempeño y el del apoyo a partir del contexto social actual. En los últimos años se ha promovido en las instituciones prestadoras de los servicios de salud la colaboración como la vía para alcanzar las metas de calidad y excelencia. ${ }^{4}$ La colaboración es deseable de incorporar y experimentar en los quehaceres cotidianos de las organizaciones con la finalidad de reducir las variaciones en la calidad de la gestión del cuidado enfermero. ${ }^{9}$

Aunque en este estudio la preferencia por el modelo del desempeño fue discreta, se entiende que no le son propicias las condiciones actuales de la cultura organizacional. Existe poco consenso entre el personal sobre las creencias, actitudes y los valores que son inherentes al modelo del desempeño, tales como la cooperación, el énfasis en el reconocimiento a los logros del equipo y la eficiencia. ${ }^{12}$

González-Pier y colaboradores ${ }^{15}$ señalan que es necesario que entre el personal de enfermería haya consenso respecto a las capacidades y las funciones para la generación e instauración de una cultura organizacional deseada, para, a su vez, fortalecer y desarrollar a las organizaciones hospitalarias públicas.

Para lograr que exista el modelo de cultura organizacional pertinente a los propósitos y aspiraciones de calidad, eficiencia y seguridad del paciente que persigue la Secretaría de Salud, hace falta poner énfasis en el desarrollo profesional del personal de enfermería, en la aplicación de prácticas basadas en evidencia científica, en la seguridad de los pacientes y en la eficiencia de la gestión de los servicios. ${ }^{1,2}$

La selección aleatoria de los participantes fortalece la confianza en que los resultados encontrados pueden repetirse en un estudio hecho en condiciones similares. La pérdida de participantes fue de $11.76 \%(\mathrm{n}=4)$. Los cuestionarios incompletos fueron eliminados y no hubo negativas a participar.

\section{Conclusiones}

Los resultados presentados en este estudio sobre la cultura organizacional en el servicio de enfermería señalan al modelo del poder como dominante y al modelo del desempeño como el preferido. Identificar estos modelos de la cultura organizacional permite entender los diferenciales de desempeño en la gestión del cuidado enfermero actual de los servicios que se brinda a los usuarios del hospital y plantearse aspiraciones de mejoras en el propio servicio.

La discrepancia entre lo que se vive en el hospital y lo que se aspira tener como modelo de cultura organizacional constituye una ventana de oportunidad para que el gremio de enfermería se oriente hacia la autonomía y la solidaridad-ambos considerados como pilares de la profesionalización- y genere sus propios estándares de gestión del cuidado enfermero.

Instaurar una cultura organizacional en los servicios de enfermería, tendiente al modelo del desempeño, puede hacerse a través de fomentar el compromiso profesional con los pares y la renuncia al reconocimiento, a la premiación por logros individuales o personales, y con la reforma de los esquemas legales actuales de la institución. Todo ello contribuiría a abrir áreas de oportunidad de mejora en otros grupos de profesionales adscritos a los hospitales tales como los de las áreas médica y administrativa.

Para que una propuesta de innovación y desarrollo en los servicios de enfermería sea exitosa requiere dirigirse hacia la implantación de la cultura del desempeño. Los resultados de este estudio muestran que la cultura organizacional en el servicio de enfermería es necesaria para mejorar la calidad de los servicios que se brindan y adicionalmente contribuir a la profesionalización del gremio.

Se sugiere profundizar en el estudio de las implicaciones que tiene el modelo dominante del poder en el servicio de enfermería en los hospitales de segundo nivel, con respecto a los propósitos y aspiraciones de calidad, eficiencia y seguridad del paciente que la Secretaría de Salud persigue. En términos organizacionales, es fundamental para los hospitales de segundo nivel 
de atención contar con la cultura que le sea favorable para alcanzar sus metas.

\section{Agradecimiento}

Los autores agradecen a las enfermeras especialistas en salud pública Mariela Aranda Sandoval y Olga Zúñiga García por su participación en el trabajo de campo.

Declaración de conflicto de intereses: Los autores declararon no tener conflicto de intereses.

\section{Referencias}

I.Arredondo A, Icaza E. Financial requirements for the treatment of diabetes in Latin America: implications for the health system and for patients in Mexico. Diabetología 2009; 52:1693-1695.

2.Arredondo A, Zúñiga A. Epidemiologic Changes and Economic Burden of Hypertension in Latin America. Am J Hypertens 2006; 19:553-559 3. Secretaría de Salud. Programa Nacional de Salud 2007-2012. México, DF: SS, 2007:148.

4. Secretaría de Salud.Acuerdo por el que la Secretaría de Salud da a conocer las Reglas de Operación 2010 del Programa Sistema Integral de Calidad en Salud (SICALIDAD). Decimocuarta sección. Diario Oficial. Martes 29 de diciembre de 2009.
5. Kurganct P, Melleiro MM, Rizatto-Tronchin DM. Indicadores para avaliação de qualidade do gerenciamento de recursos humanos em enfermagem. Rev Bras Enferm Brasília 2008;6 I(5):539-544.

6. Chiavenato I.Administración de recursos humanos. 4a ed. México, DF: Mc Graw- Hill, 2000:589.

7. Instituto Mexicano del Seguro Social. La Cultura Organizacional en los Sistemas de Salud. Rev Med IMSS 2002;40(3):203.

8. Ng TWH, Sorensen KL,Yim F. Does the job satisfaction-job performance relationship vary across cultures? J Cross Cult Psychol 2009;40(5):761-796.

9. Schepers R. Regulation and Trust in Action:The Subtle Balance between Doctors and Management in Two Belgian Hospitals. Curr Sociol 2006;54: 637

10. Hampton D.Administración. $3^{3}$ ed. México, DF: Mc Graw Hill, 1990:473.

II. Sorensen JB. The Strength of Corporate Culture and the Reliability of Firm Performance. Adm Sci Q 2002;47:70.

12. Hartasánchez-Garaña JM. Perfil dinámico de la cultura organizacional de los directivos públicos en México.VII Congreso Internacional del CLAD sobre la Reforma del Estado y de la Administración Pública, Lisboa, Portugal, 8-II Oct. 2002:20.

I3.Alemán-Escobar ML, Molina-Rodríguez JF. Cultura organizacional de servicios de enfermería. Desarrollo Cientif Enferm 2005; I3(3):68-72. 14. de Carvalho-Jericó M, Maris-Pérez A, Kurcgant P. Organizational structure of nursing services: reflections on the influence of the organizational power and culture. Rev esc enferm USP 2008;42(3):569-577. 15. González-Pier E, Gutiérrez-Delgado C, Stevens G, Barraza-Lloréns M, Porras-Condey R, Carvalho N, et al. Definición de prioridades para las intervenciones de salud en el Sistema de Protección Social en Salud de México. Salud Publica Mex 2007;49 supl I:S37-S52. 